\title{
Far-Ultraviolet Observations of Outflows from Infrared-Luminous Galaxies
}

\author{
Claus Leitherer ${ }^{1}$, Rupali Chandar ${ }^{2}$, Christy A. Tremonti ${ }^{3}$, and \\ Aida Wofford ${ }^{1}$ \\ ${ }^{1}$ Space Telescope Science Institute, 3700 San Martin Dr., Baltimore, MD 21218, USA \\ email: leitherer@stsci.edu, wofford@stsci.edu \\ ${ }^{2}$ The University of Toledo, Dept. of Physics and Astronomy, Toledo, OH 43606, USA \\ email: rupali.chandar@utoledo.edu \\ ${ }^{3}$ University of Wisconsin, Dept. of Astronomy, 475 N. Charter St., Madison, WI 53706, USA \\ email: tremonti@astro.wisc.edu
}

\begin{abstract}
We have obtained ultraviolet spectra between 1150 and $1450 \AA$ of four ultravioletbright, infrared-luminous starburst galaxies. Our selected sight-lines towards the starburst nuclei probe the conditions in the starburst-driven outflows. We detect outflowing gas with velocities of up to $\sim 900 \mathrm{~km} \mathrm{~s}^{-1}$. It is likely that the outflows are a major source of metal enrichment of the galaxies' halos. The mass outflow rates of several tens of $\mathrm{M}_{\odot} \mathrm{yr}^{-1}$ are similar to the starformation rates. The outflows may quench star formation and ultimately regulate the starburst.
\end{abstract}

Keywords. galaxies: halos — intergalactic medium — galaxies: starburst — ultraviolet: ISM

\section{Introduction}

The absorption spectra of quasi-stellar objects demonstrate that metals in the universe are found far from their production sites. Mg II absorbers are associated with galaxies at impact parameters well outside their luminous dimensions. Advances in ground- and space-based telescopes and detectors have pushed the discovery space to many other ions, as well as to low and high redshift (e.g., Ménard et al. 2011; Tumlinson et al. 2011a).

An alternative technique of probing the circumgalactic gas is to directly target galaxies and measure the self-absorption in their spectra. Absorption-line studies of circumgalactic gas have been performed at low (e.g., Rupke, Veilleux, \& Sanders 2005a,b,c; Chen et al. 2010), intermediate (e.g., Weiner et al. 2009; Coil et al. 2011; Erb et al. 2012), and high (e.g., Pettini et al. 2002; Steidel et al. 2010) redshift.

Starburst galaxies are of particular interest. Galactic-scale outflows of gas are ubiquitous in the most actively star-forming galaxies in the local (Chen et al. 2010) and distant universe (Shapley et al. 2003). These outflows affect the evolution of galaxies and the intergalactic medium (Veilleux, Cecil, \& Bland-Hawthorn 2005). For example, by selectively blowing metals out of shallow galactic potential wells, they may explain the tight relation between stellar mass and metallicity (Tremonti et al. 2004).

The ultraviolet (UV) is key for understanding starbursts. Previous space-UV spectroscopy has focused almost exclusively on blue, and therefore metal-poor, low-luminosity starburst galaxies but has neglected the powerful, infrared-(IR) bright (but UV-faint) nuclear starbursts. Yet, IR-bright starbursts may have very different properties in terms of their initial mass function (IMF), specific star-formation intensity, and their relation to the circumgalactic gas. We have obtained UV spectroscopy of four IR-luminous starburst galaxies whose UV brightness makes them the targets of choice for this purpose. 
Table 1. Galaxy properties.

\begin{tabular}{|c|c|c|c|c|c|}
\hline Galaxy & $\begin{array}{c}v_{\text {helio }} \\
{\left[\mathrm{km} \mathrm{s}^{-1}\right]}\end{array}$ & $\begin{array}{c}\log \left(L_{\mathrm{IR}}\right) \\
{\left[\mathrm{L}_{\odot}\right]}\end{array}$ & $12+\log (\mathrm{O} / \mathrm{H})$ & $\begin{array}{c}E(B-V) \\
{[\mathrm{mag}]}\end{array}$ & $\begin{array}{c}\mathrm{SFR}_{\mathrm{UV}} \\
{\left[\mathrm{M}_{\left.\odot \mathrm{yr}^{-1}\right]}\right.}\end{array}$ \\
\hline F $08339+6517$ & 5730 & 11.1 & 8.45 & 0.34 & 70 \\
\hline NGC 3256 & 2804 & 11.6 & 8.73 & 0.54 & 55 \\
\hline NGC 6090 & 8785 & 11.6 & 8.77 & 0.28 & 29 \\
\hline NGC 7552 & 1608 & 11.1 & 8.74 & 0.54 & 57 \\
\hline
\end{tabular}

\section{Sample}

Naively, one would expect dusty, IR-luminous galaxies to be dauntingly faint in the UV. However, much of the dust responsible for the UV extinction is apparently distributed around the starburst in the form of a moderately inhomogeneous foreground screen (Calzetti 2001). Therefore we are able to directly study much of the stellar population responsible for powering the starburst via observations in the UV.

The selected galaxies are F08339+6517, NGC 3256, NGC 6090, and NGC 7552. No strong AGN activity has been detected in any of the galaxies. A summary of some basic properties of the galaxies is in Table 1 . Their heliocentric velocities $\left(v_{\text {helio }}\right)$ are large enough to preclude blending gas absorption lines from the target galaxy and the Milky Way.

We used the G130M mode of HST's COS. The four galaxies are nuclear starbursts, and the $2.5^{\prime \prime}$ circular COS aperture (projected size $\sim 300$ and $1600 \mathrm{pc}$ ) was placed on the galaxy centers. We corrected the spectra for foreground reddening and converted to restframe wavelengths. We measured the widths of the Milky Way foreground lines, which are unresolved at the nominal COS G130M resolution of $\mathrm{R}=17,000$. Typical widths are $\sim 0.5 \AA$. In Figure 1 we show the rectified spectrum of F08339+6517 as an example. We identified the major spectral features using the line list of Leitherer et al. (2011).

\section{Internal Reddening and Stellar Populations}

We applied the standard technique of UV spectral synthesis (e.g., Wofford, Leitherer, \& Chandar 2011) to constrain the most recent star-formation history traced by massive OB
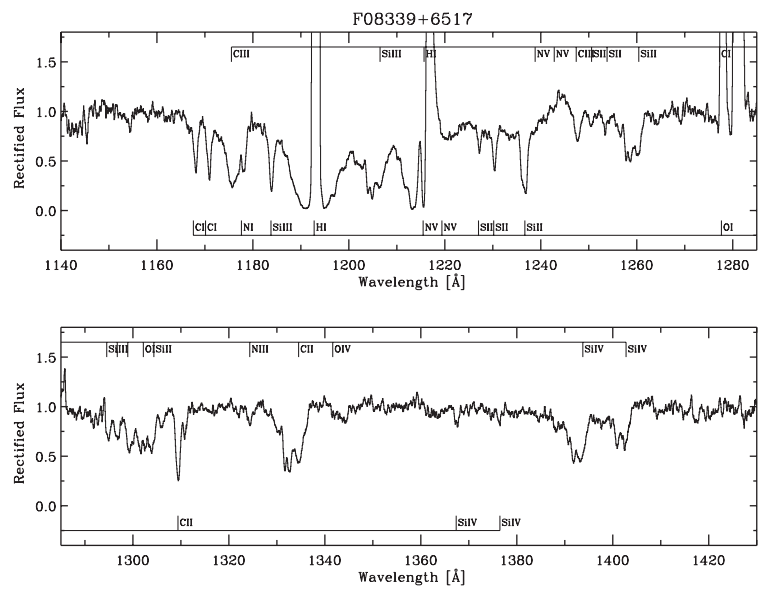

Figure 1. Rectified COS spectrum of F08339+6517. The wavelength scale is in the galaxy restframe. Milky Way lines are identified below the spectrum; galaxy lines are above. 
stars. The intrinsic reddening was determined by comparing the observed UV continuum slope to the one predicted by the models. In column 5 of Table 1 we report the derived total reddening $E(B-V)$, which is the sum of the foreground and intrinsic reddening.

After dereddening the spectra with a Calzetti (2001) attenuation curve, the monochromatic UV luminosities were obtained. By extrapolating to lower masses using the adopted IMF and correcting for aperture-size effects, we derived the total star-formation rates (SFR) listed in column 6 . SFR ranges between 30 and $70 \mathrm{M}_{\odot} \mathrm{yr}^{-1}$, which are typical values for IR-luminous galaxies. All four galaxies follow the empirical relation between the far-IR luminosity and the UV reddening (Meurer, Heckman, \& Calzetti 1999).

\section{Outflow Properties}

Lyman- $\alpha$ is the strongest emission line in all four galaxies. The equivalent widths range from 2 to $13 \AA$, which can be compared to the value of $\sim 100 \AA$ expected for a 20 Myr old continuous starburst under Case B conditions. This suggests a Lyman- $\alpha$ escape fraction of $\sim 10 \%$. The escape probability of Lyman- $\alpha$ photons strongly depends on the velocity structure of the ISM and the dust content (Kunth et al. 1998; Charlot \& Fall 1993). Although our sample is small, we note the absence of any correlation with reddening.

Schaerer et al. (2011) generated a library of theoretical Lyman- $\alpha$ profiles formed in the moving media of star-forming galaxies. These profiles are useful for determining the column densities, kinematics, and dust content of the neutral ISM along the sight-lines to the nuclear starbursts. Comparison with the observed profiles suggests H I column densities of order $10^{20} \mathrm{~cm}^{-2}$.

The strongest absorption features are the low-ionization lines of Si III $\lambda 1206$, Si II $\lambda 1260$, and C II $\lambda 1334$. Inspection of the line profiles suggests a complex structure with several components present. We used the Galactic foreground lines as fiducials to verify that the observed profiles are not affected by artifacts. The profiles are blueshifted and composed of several discrete components with velocities of up to $\sim 900 \mathrm{~km} \mathrm{~s}^{-1}$. The bulk
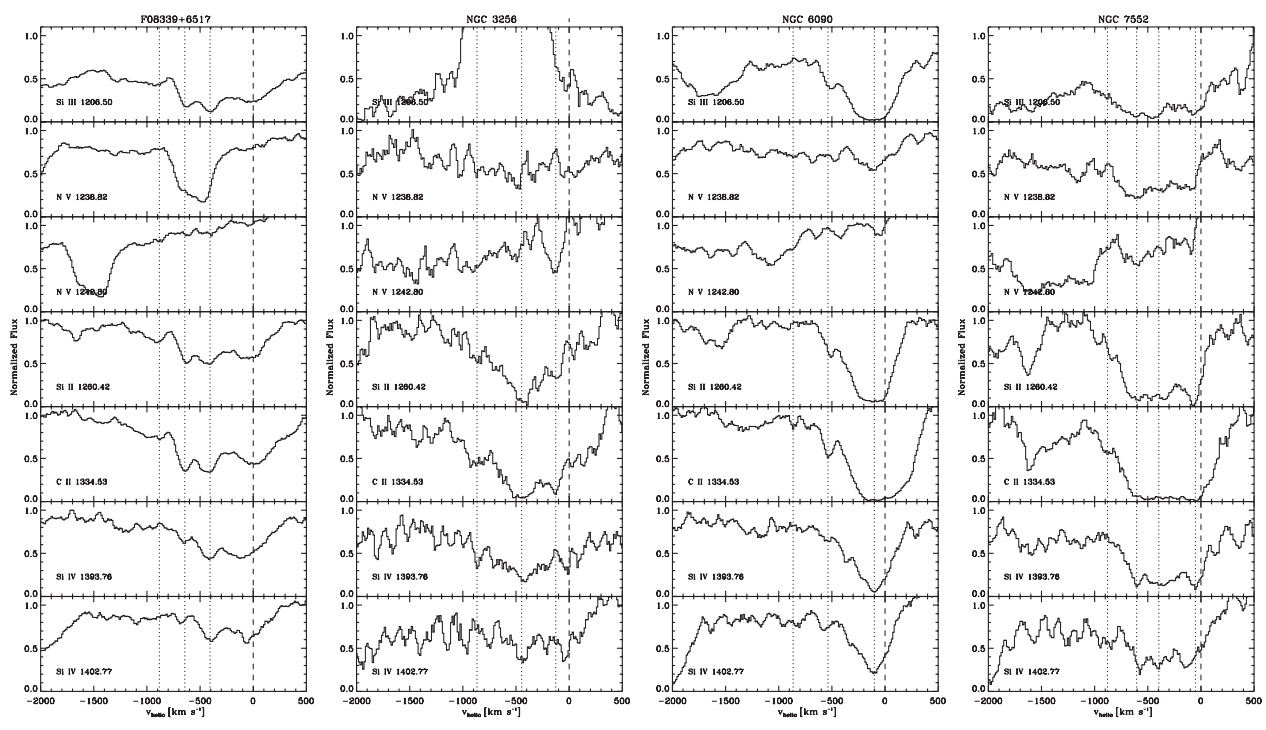

Figure 2. Continuum-normalized absorption profiles versus $v_{\text {helio }}$ for F08339+6517, NGC 3256, NGC 6090, and NGC 7552 (from left to right). The lines are from top to bottom: Si III $\lambda 1206$, Si II $\lambda 1260$, C II $\lambda 1334$, Si IV $\lambda 1393$, and Si IV $\lambda 1402$. The dashed vertical line indicates zero velocity. Dotted vertical lines indicate discrete components at velocities up to $\sim 900 \mathrm{~km} \mathrm{~s}^{-1}$. 
velocities are around $500 \mathrm{~km} \mathrm{~s}^{-1}$. This is illustrated in Figure 2, where we plot Si III, Si II, C II and other lines (discussed below) in velocity space with the detected components highlighted. We chose a conservative approach and dismissed structures whose origin could not clearly be attributed to a high-velocity component in all three lines. For instance a strong absorption at $1324 \AA$ is more likely due to N III $\lambda 1324.35$ rather than blueshifted C II. Given the redshift of the four galaxies, we can confidently rule out any confusion with Galactic high-velocity clouds. Higher-ionization lines, such as Si IV $\lambda 1400$ and N V $\lambda 1240$ show P Cygni profiles due to stellar winds. The wind signatures preclude firm conclusions on the presence of the features detected in the low-ionization lines.

The most natural interpretation of the observed velocity structure is expanding warm $\left(\sim 10^{4} \mathrm{~K}\right)$ gas driven by the current burst of star formation. The individual components indicate density inhomogeneities of the outflow, as expected for interstellar material entrained in a galactic-scale wind. The flow time scale of $\sim 10 \mathrm{Myr}$ (for an assumed size of $5 \mathrm{kpc}$ and $v=500 \mathrm{~km} \mathrm{~s}^{-1}$ ) corresponds to the typical duration of starbursts. Does the material escape from the gravitational well of the galaxies? The observed maximum outflow velocities of the four galaxies are similar to the escape velocities for an assumed halo extending to $100 \mathrm{kpc}$. Therefore it is not likely that the material will escape from the galaxy potential. Instead it may accumulate in the halo, which serves as a reservoir for newly formed metals (Tumlinson et al. 2011b).

Following Heckman et al. (2000), Rupke et al. (2005a,b,c), and Weiner et al. (2009), we can estimate the mass outflow rates from the observed $\mathrm{H}$ I columns, the characteristic length scale, and the outflow velocities: $\dot{M}=22 \mathrm{M}_{\odot} \mathrm{yr}^{-1} \frac{N_{\mathrm{H}}}{10^{20} \mathrm{~cm}^{-2}} \frac{R}{5 \mathrm{kpc}^{2}} \frac{v}{300 \mathrm{~km} \mathrm{~s}^{-1}}$. The rates of $10^{1}-10^{2} \mathrm{M}_{\odot} \mathrm{yr}^{-1}$ are similar to the star-formation rates. Therefore, the outflows may quench star formation and ultimately regulate the starburst.

\section{References}

Calzetti, D. 2001, PASP, 113, 1449

Charlot, S. \& Fall, S. M. 1993, ApJ, 415, 580

Chen, Y-M., Tremonti, C. A., Heckman, T. M., et al. 2010, AJ, 140, 445

Coil, A. L., Weiner, B. J., Holz, D. E., et al. 2011, ApJ, 743, 46

Heckman, T. M., Lehnert, M. D., Strickland, D. K., \& Armus, L. 2000, ApJS, 129, 493

Erb, D. K., Quider, A. M., Henry, A. L., \& Martin, C. L. 2012, arXiv:1209.4903

Kunth, D., Mas-Hesse, J. M., Terlevich, E., et al. 1998, A\&\&A, 334, 11

Leitherer, C., Tremonti, C. A., Heckman, T. M., \& Calzetti, D. 2011, AJ, 141, 37

Ménard, B., Wild, V., Nestor, D., et al. 2011, MNRAS, 417, 801

Meurer, G. R., Heckman, T. M., \& Calzetti, D. 1999, ApJ, 521, 64

Pettini, M., Rix, S. A., Steidel, C. C., Adelberger, K. L., Hunt, M. P., \& Shapley, A. E. 2002, ApJ, 569, 742

Rupke, D. S., Veilleux, S., \& Sanders, D. B. 2005a, ApJS, 160, 87

- 2005b, ApJS, 160, 115

- 2005c, ApJ, 632, 751

Schaerer, D., Hayes, M., Verhamme, A., \& Teyssier, R. 2011, A\&A, 531, A12

Shapley, A. E., Steidel, C. C., Pettini, M., \& Adelberger, K. L. 2003, ApJ, 588, 65

Steidel, C. C., Erb, D. K., Shapley, A. E., et al. 2010, ApJ, 717, 289

Tremonti, C. A., Heckman, T. M., Kauffmann, G., et al. 2004, ApJ, 613, 898

Tumlinson, J., Thom, C., Werk, J. K., et al. 2011b, Science, 334, 948

Tumlinson, J., Werk, J. K., Thom, C., et al. 2011a, ApJ, 733, 111

Veilleux, S., Cecil, G., \& Bland-Hawthorn, J. 2005, ARA\&A, 43, 769

Weiner, B. J., Coil, A. L., Prochaska, J. X., et al. 2009, ApJ, 692, 187

Wofford, A., Leitherer, C., \& Chandar, R. 2011, ApJ, 727, 100 